\title{
Integration of Environmental Information in a Mining Region Using a Geoportal
}

\author{
Svetlana Ivanova ${ }^{1}$, Elena Sant'eva ${ }^{1}$, Maxim Bakanov ${ }^{1}$, Leszek Sobik ${ }^{2}$, and Leonid \\ Lopukhinsky $^{3}$ \\ ${ }^{1}$ Kemerovo State University, 650000, 6 Krasnaya st., Kemerovo, Russia \\ ${ }^{2}$ Polska Grupa Górnicza S.A., KWK ROW ruch Chwałowice, ul. Przewozowa 4, 44-206 Rybnik, \\ Poland \\ ${ }^{3}$ T.F. Gorbachev Kuzbass State Technical University, Mezhdurechensk Branch, 652881 \\ Mezhdurechensk, 36 Stroiteley st., Russian Federation
}

\begin{abstract}
At present, the complex nature of the impact on the ecosystem in regions with intensive mining creates a multidimensional information "plume" consisting of data on mineral reserves, the state of mining operations, accumulated, current and future environmental pollution. The transition to the lean use of the subsoil and the reasonable disposal of mining waste requires fundamentally new forms of environmental information accumulation and processing during designing new enterprises and regulating the activities of existing ones. The most promising form of information support for the greening of mining is a geoportal. It is a complex of software and technological support for working with spatial data. Its key task is to provide the users with tools and services for storing and cataloging, publishing and loading spatial and environmental data, searching and filtering by metadata, interactive web visualization, direct access to geodata based on map web services.
\end{abstract}

\section{Introduction}

Today, when geographic information systems (GIS) have evolved from exotic tools for processing spatial information into working tools, therefore their evolution is taking place in several directions [1-3]:

- development of applied information and reference systems, including methods of working with electronic maps, which involves carrying out the simplest geometric calculations, determining the coordinates of objects of interest, the possibility of calculating their areas or perimeters, laying routes, printing maps, constructing various diagrams reflecting the distribution of one or another indicator;

- creation of geo-computing systems based on complex mathematical models and involving cartographic information to set the initial and boundary conditions, calculation parameters, followed by displaying the results on the map;

- creation of geoportals that provide users with access to certain resources, including Web applications and specific types of services; 
- development of systems based on the principles of artificial intelligence and assuming automated processing of spatial heterogeneous information to obtain new knowledge and rules.

The development of cloud technologies and artificial intelligence systems today makes it possible to combine these different directions within geoportals that ensure the integration of heterogeneous data and computational manipulations with them, allowing the creation of fundamentally new information and computing systems that provide the user with the necessary knowledge, types of services in selected area.

\section{Materials and Methods}

The INSPIRE directive defines a geoportal through a set of its functions as a site or its equivalent, the list of functions of which, implemented in the form of Web services (geoservices), includes the search for datasets, their visualization (geo-visualization), loading and transformation, as well as calling other services. In our understanding, a geoportal is defined as some specialized site that uses most types of cloud services, which allow not only to provide information search and its subsequent visualization, but also to carry out complex mathematical calculations, generate and update storages of heterogeneous geodata, including state of mining works, obtain new knowledge by means of artificial intelligence systems, including datamining and cognitive systems [4].

This approach to the construction of geoportals allows you to focus on the tasks being solved and to apply all the variety of modern approaches to the integrated processing of spatial heterogeneous information [5].

The use of cloud services, on the one hand, simplifies the solution of many issues, allowing not to focus on specific sources of geoinformation, but to define them through a service (DAAS-data as service), which provides the ability to work with them. At the same time, it turns out to be quite simple to create specific geoportals based on the so-called MASHUPS interfaces, which are a set of basic modules for working with cartographic and other spatial information [4]. The advantage of this approach include:

- fast creation of new dynamic applications;

- simplicity, realized through the integration of sources;

- further promotion of scientific services through minimizing data management;

- reduction of efforts for application development with the ability to quickly adapt to new situations;

- fast acquisition of distributed data.

The general scheme of operation of MASHUPS interfaces is shown in Fig. 1. 


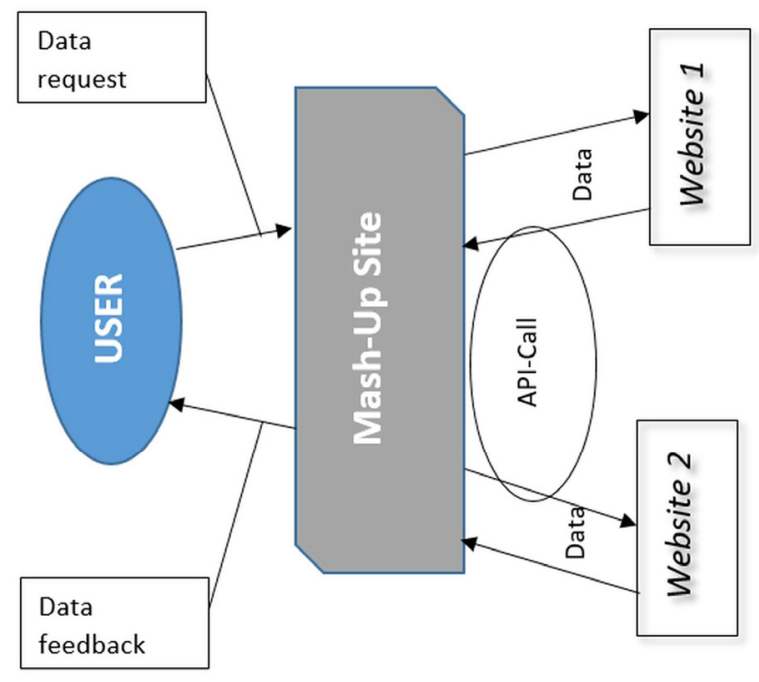

Fig. 1. Scheme of operation of MASHUPS interfaces.

Further development of MASHUPS technology involves [4]:

- transition from individual solutions to platforms for the integration of a variety of dynamic sources;

- development of semantics in the direction of taxonomy, the creation of new types of metadata, controlled vocabularies, the transition to natural categories;

- expansion of infrastructure, integration of content with minimal technological elements (XML);

- deep integration with artificial intelligence systems: knowledge is not just data;

- combining semantic framework to include unstructured content in MASHUPS.

Focusing on cloud computing as one of the main elements of geoportals, we consider from the whole set of services only those that are naturally enough integrated into specific solutions.

The cloud service SAAS (Software as a Service) assumes the presentation of certain software, including specific basic GIS and calculation systems, which can be accessed by means of MASHUPS. To implement this type of service, it is necessary to have appropriate catalogs of software available (including on the Internet), corresponding meta descriptions of geodata, and some internal standard for working with this type of service. DAAS (Data as Service) is a method for obtaining various geodata, including those received from calculations. This type of service can also include obtaining geodata, including images already processed according to certain algorithms. It is especially important for hyperspectral, multispectral and radar data, since their thematic processing can only be performed by professionally trained specialists. At the same time, the user is freed from complex and computationally expensive processing and receives a guaranteed result. For this type of service, a crowdsourcing approach can be applied, when the user provides his own unique data, and in return receives processing or analysis data [6].

PAAS (Platform as Service) is a service associated with the presentation of middleware for the implementation of specific geosciences. Today, when geo-computing systems require more and more computing power, the issue of parallelizing tasks and computational models, their implementation on cluster-type systems or on GRID systems is on the agenda. In addition, this is where this type of service is required, providing a remote solution to specific tasks. 
IAAS (Infrastructure as Service) is the most time-consuming and technically complex type of service, including hardware, computer computing and data storage technologies, operating systems, which are presented not as local resources, but through accessing services hosted on the provider's side. A special case is Hardware as Service (HAAS). In this case, the implementation of a geoportal requires significant efforts, usually federal level geoportals created within the framework of the corresponding telecommunication structures.

\section{Results and Discussion}

The types of cloud services we have considered by no means exhaust the possible schemes for constructing geoportals, but represent the most used models of cloud systems.

Any geoportal is determined by the set of functions that it is capable of supporting and the balance between them. Today there are geoportals of various subject specialization and departmental affiliation - from thematically universal to special ones, from federal to local ones [7-8]. Respectively, when creating a specific system, one must proceed from the tasks that it is intended to solve. The approach we propose is quite versatile and has already been tested in the implementation of several systems.

Next, let us dwell on the use of data storages in geoportals as a new element of the system that integrates and manipulates heterogeneous data, including remote sensing data, which require special processing methods. The term "data storage" refers to a special type of database that acts as a central repository for corporate data [9]. In other words, it is an archive that is integrated from operational databases and is used primarily for intelligent data processing and geo-computing systems.

Based on the above elements, an environmental safety monitoring system was created as a distributed information and computing environment using elements of a cloud service [10].

A cloud service is a special client-and-server technology, where a client uses resources (processor, RAM, disk space, network channels, specialized controllers, software, etc.) of a group of servers in a network interacting in such a way that:

-for the client, the whole group looks like a single virtual server;

- the client can transparently and with high flexibility change the amount of consumed resources in the event of a change in his needs (increase / decrease the capacity of the server with a corresponding change in the payment for it).

At the same time, the presence of several sources of used resources, on the one hand, makes it possible to increase the availability of the client-and-server system due to the possibility of scaling with an increase in the load (an increase in the number of sources in proportion to an increase in demand for it and / or transfer of a working virtual server to a more powerful source, "live migration"). On the other hand, it reduces the risk of a virtual server inoperability in the event of failure of any of the servers included in the client group, since instead of a failed server, it is possible to automatically reconnect a virtual server to the resources of another (backup) server.

The technological core of the system is the Google App Engine environment, which allows you to create web applications using standard Java technologies and run them on the scalable Google infrastructure. The Java environment is a Java 6 virtual machine (JVM), a Java servlet interface, and support for standard interfaces for scalable data storage and App Engine services such as JDO, JPA, JavaMail, and JCache [4]. Standards support simplifies application development and makes it easy to port your application both from and into your own servlet environment.

Free software is used as development tools, which add new project wizards and debug configurations to the IDE for App Engine projects. App Engine for Java greatly simplifies the development and deployment of international web applications. The Eclipse plugin is bundled with the App Engine. App Engine runs Java applications using the Java 6 Virtual 
Machine (JVM). The App Engine SDK supports Java 5 or later. The Java 6 JVM can also use classes compiled with a compiler of any version up to Java 6. App Engine is used for web applications, which are the glue of all system components. App Engine executes requests by invoking servlets according to the deployment descriptor. The JVM operates in a secure environment, which allows the application to be isolated for maintenance and security purposes. The environment ensures that applications can only perform actions that do not affect the performance and scalability of other applications.

Authentication service gives secure access to confidential information, which is provided by means of the Google Users API. The Google App Engine platform has tight integration with Google Accounts: applications can allow users to log in using their existing Google accounts. With the help of Google accounts, users will be able to get started with the application faster without having to register specifically on the site once, and the developer will be able to personalize the application. App Engine applications can authorize their users using Google accounts. The app can redirect the user to the login and registration page, or let them log out of the account. After the user is authorized, the application can access his email address and the specified alias. You can also determine if the user is an application administrator and has access to restricted areas of the site, which will allow you to create the system.

The mapping service provides the ability to visualize subject information on backing maps, including Google. It is programmatically divided into two parts: public and private. In the public domain, the Google Maps API technology is used, which provides an open interface of functions for working with Google maps. This functionality is used for cartographic calculations and coordinate referencing of classified information both from this service and from database services and settlement services. The technology allows you to associate Google maps with a specific website using a unique API key. API objects contain methods for loading third-party maps in KML/KMZ format. KML / KMZ-resources are hosted on the Apache web server.

The system of geoportal for mining regions must develop and present the following thematic maps:

- monitoring of surface waters;

- monitoring of underground waters;

- monitoring of snow cover;

- types of landscapes (combined with vegetation cover);

- soil types;

- disturbed lands;

- objects of emissions of pollutants into the atmospherez4

- rare and endangered species of vegetation.

Calculation services contain the program logic of mathematical models of the subject area, implemented on the basis of RPC technologies, developed as Java servlets, which allows them to be integrated with any of the above system services. Such a geoportal system implements models for assessing emissions and distribution of pollutants into the atmosphere; fallout of industrial aerosols onto the underlying surface; the spread of pollutants and their fallout to the surface during industrial explosions, taking into account the real wind rose; assessing the quality of surface and ground waters, shaking and noise impact during massive explosions.

\section{Conclusion}

Thus, the prototype of a geoportal - integrated information and computing system for dynamic assessment of the ecological state of a coal-mining area includes the following. 
The system consists of a cartographic module, a database module, a calculation module and an authentication module. A cloud service is a special client-and-server technology - the use by a client of a group of servers in a network interacting in such a way that:

-for the client, the whole group looks like a single virtual server;

- the client can transparently and with high flexibility change the amount of consumed resources in the event of a change in his needs.

The system provides collection and storage of data on the monitoring of natural resources, assessment and forecast of the geo-ecological state of the territory adjacent to the mining (land) allotment of a coal-mining (coal-processing) enterprise, as well as a coal-mining region, on the territory of which enterprises of various profiles can be located. The system integrates and provides information support for both ground monitoring data and remote monitoring data. The developed system is a standard for information support for monitoring the environment and industrial activity of a coal-mining (coal-processing) enterprise and a coal mining region [11].

\section{References}

1. I. Kudryashova M. Venger, N. Zakharova, E3S Web Conf. 105, 02005 (2019)

2. E. Shavina, V. Prokofev, E3S Web of Conf. 174, 04017 (2020)

3. M. Tyulenev, S. Zhironkin, E. Tyuleneva, A. Abay, S. Anyona, M. Hellmer, Coal International 265(3), 30-34 (2017)

4. V.A. Kovalev, V.P. Potapov, E.L. Schastlivtsev, Yu.I. Shokin, Modeling of geoecological systems of coal mining regions (SB RAS, Novosibirsk, 2015)

5. I. Kudryashova, N. Zakharova, E. Kharlampenkov, E3S Web Conf. 21, 02016 (2017)

6. E.A. Taran, V.A. Malanina, F. Casati, Economics and Innovation Management 4, 39-46 (2020) DOI: 10.26730/2587-5574-2020-4-39-46

7. T.V. Kiseleva, V.G. Mikhailov, Economics and Innovation Management 4, 70-78 (2018) DOI: 10.26730/2587-5574-2018-4-70-78

8. F.N. Abu-Abed, L.G. Naumova, Journal of Mining and Geotechnical Engineering 3, 2135 (2019) doi: 10.26730/2618-7434-2019-3-21-35

9. V.V. Guzyr', Economics and Innovation Management 4, 4-19 (2019) DOI: 10.26730/2587-5574-2019-4-4-19

10. F. Abu-Abed, E3S Web Conf. 105, 03006 (2019)

11. E. Shavina, V. Prokofev, E3S Web Conf. 174, 02014 (2020) 